\title{
Ultrarelativistic circular orbits of spinning particles in a Schwarzschild field
}

\author{
Roman Plyatsko \\ Pidstryhach Institute of Applied Problems in Mechanics and Mathematics \\ Ukrainian National Academy of Sciences, 3-b Naukova Str., \\ Lviv, 79060, Ukraine \\ E-mail: plyatsko@lms.lviv.ua
}

\begin{abstract}
.
Ultrarelativistic circular orbits of spinning particles in a Schwarzschild field described by the Mathisson-Papapetrou equations are considered. The preliminary estimates of the possible synchrotron electromagnetic radiation of highly relativistic protons and electrons on these orbits in the gravitational field of a black hole are presented
\end{abstract}

Submitted to: Class. Quantum Grav.

PACS numbers: $0420,9530 \mathrm{~S}$ 


\section{Introduction}

The theory of synchrotron radiation of different types (electromagnetic, scalar, gravitational) near a Schwarzschild and Kerr black hole was initiated more than 30 years ago 1, 2]. Two cases of the synchrotron radiation are distinguished: 1) when a test particle is moving in a highly relativistic geodesic circular orbit about a black hole, and 2) when an orbit is circular but nongeodesic, caused by the combined effect of the gravitational field and other factors (for example, a magnetic field). In a Schwarzschild field the first case is realized on the ultrarelativistic circular orbits of the radius $r=3 m(1+\delta), \quad 0<\delta \ll 1$ ( $m$ is the Schwarzschild mass; in the system of units used here, the velocity of light in vacuum and the gravitational constant are equal to 1$)$.

The purpose of this paper is to draw attention to a possible case of the nongeodesic synchrotron radiation caused by the particle's spin interacting with the gravitational field. That is, when in addition to the gravitational no other fields or factors are required. We hope that our investigation will be of interest both in the context of the recent investigations of the gravitational radiation of a spinning particle near black holes 3. 4, 5], as well as for general estimates of the nongravitational synchrotron radiation 6].

We shall use the Mathisson-Papapetrou equations describing the motion of a spinning test particle (body) in a gravitational field [7, 8]

$$
\begin{aligned}
& \frac{D}{d s}\left(M u^{\lambda}+u_{\mu} \frac{D S^{\lambda \mu}}{d s}\right)=-\frac{1}{2} u^{\pi} S^{\rho \sigma} R_{\pi \rho \sigma}^{\lambda}, \\
& \frac{D S^{\mu \nu}}{d s}+u^{\mu} u_{\sigma} \frac{D S^{\nu \sigma}}{d s}-u^{\nu} u_{\sigma} \frac{D S^{\mu \sigma}}{d s}=0,
\end{aligned}
$$

where $u^{\lambda}$ is the 4 -velocity of a spinning particle, $S^{\mu \nu}$ is the tensor of spin, $M$ and $D / d s$ are, respectively, the mass and the covariant derivative (here and in the following, greek indices run 1, 2, 3, 4 and latin indices 1, 2, 3; the signature of the metric $(-,-,-,+)$ is chosen). For the description of the center of mass of the test particle, equations (11), (2) are supplemented by some relationship. Because the correct definition of the center of mass for a spinning particle is a subject of discussion, different relationships (conditions) are used. Mainly the relationship [7, 9]

$$
S^{\mu \nu} u_{\nu}=0
$$

or [10, 11, 12]

$$
S^{\mu \nu} P_{\nu}=0
$$

are considered where

$$
P^{\nu}=M u^{\nu}+u_{\mu} \frac{D S^{\nu \mu}}{d s}
$$

(the corresponding references can be found in [13]).

It is shown that equations (11), (2) are the classical limit of the Dirac equation in a gravitational field [14]. 


\section{Circular orbits of highly relativistic spinning particles in a Schwarzschild field}

We begin by considering equations (11)-(3). By equation (3) the components $S^{i 4}$ can be expressed through $S^{i 4}$ :

$$
S^{i 4}=\frac{u_{k}}{u_{4}} S^{k i}
$$

Using (6), the components $S^{i 4}$ can be eliminated from equations (11), (21). Instead of three independent components $S^{i k}$ (the tensor $S^{\mu \nu}$ is antisymmetric in $\mu, \nu$ ) it is appropriate to use their linear combinations

$$
S_{i}=\frac{1}{2} \sqrt{-g} \varepsilon_{i k l} S^{k l}
$$

where $g$ is the determinant of the metric tensor, $\varepsilon_{i k l}$ is the spatial Levi-Civita symbol. The direct calculation shows that the 3-component value $S_{i}$ has the 3 -vector properties relative to the coordinate transformations $x^{\prime i}=x^{\prime}\left(x^{1}, x^{2}, x^{3}\right), \quad x^{\prime 4}=x^{4}$. The similar spin 3-vector was used in [15, 16] for the concrete metric. In many papers the 4-vector of spin $s_{\lambda}$ is considered where

$$
s_{\lambda}=\frac{1}{2} \sqrt{-g} \varepsilon_{\lambda \mu \nu \sigma} u^{\mu} S^{\nu \sigma}
$$

$\left(\varepsilon_{\lambda \mu \nu \sigma}\right.$ is the Levi-Civita symbol). By equations (66)-(8) we have the relationship between $S_{i}$ and $s_{\lambda}$

$$
S_{i}=u_{i} s_{4}-u_{4} s_{i}
$$

Using relationships (3), (17) three independent equations from (2) may be written in the form

$$
u_{4} \dot{S}_{i}-\dot{u}_{4} S_{i}+2\left(\dot{u}_{[4} u_{i]}-u^{\pi} u_{\rho} \Gamma_{\pi[4}^{\rho} u_{i]}\right) S_{k} u^{k}+2 S_{n} \Gamma_{\pi[4}^{n} u_{i]} u^{\pi}=0
$$

where a dot denotes differentiation with respect to the proper time $s$, and square brackets denote antisymmetrization of indices; $\Gamma_{\pi 4}^{\rho}$ are the Christoffel symbols. (It is known that among six equations from (2) there are only three independent equations).

Let us consider (9) for the Schwarzschild metric using the standard coordinates $x^{1}=r, \quad x^{2}=\theta, \quad x^{3}=\varphi, \quad x^{4}=t$. It is easy to check that three equations from (9) have a partial solution with $\theta=\pi / 2, \quad S_{1} \equiv S_{r}=0, \quad S_{3} \equiv S_{\varphi}=0$ and the relationship for the nonzero component of the spin 3 -vector $S_{2} \equiv S_{\theta}$ is

$$
S_{2}=r u_{4} S_{0}
$$

where $S_{0}$ is the constant of integration. The physical meaning of this constant is the same as in the general integral of the Mathisson-Papapetrou equations [17]

$$
S_{0}^{2}=\frac{1}{2} S_{\mu \nu} S^{\mu \nu}
$$

We stress that relationship (10) is valid for any equatorial motions $(\theta=\pi / 2)$ when spin is orthogonal to the motion plane $\left(S_{k} u^{k}=0\right)$.

The possible equatorial orbits of a spinning particle are described by equation (11). In the following we shall confine ourselves to the case of the circular orbits. 
For the investigation of conditions of existence of equatorial circular orbits of a spinning particle in a Schwarzschild field we shall use equations (11), (10) and the general relationship for the velocity components $u_{\mu} u^{\nu}=1$. From the geodesic equations in this field follow the algebraic relationships determining the dependence of the velocity of a particle without spin on the radius of the circular orbit. Similarly, from equation (11) we obtain the relationship for the equatorial circular orbits of a spinning particle in a Schwarzschild field

$$
\begin{aligned}
& u_{\perp}^{3} \beta\left(1-\frac{3 m}{r}\right)^{2}-u_{\perp}^{2}\left(1-\frac{2 m}{r}\right)\left(1-\frac{3 m}{r}\right) \\
& +u_{\perp} \beta \frac{m}{r}\left(2-\frac{3 m}{r}\right)+\frac{m}{r}\left(1-\frac{2 m}{r}\right)=0
\end{aligned}
$$

where $u_{\perp}=r \dot{\varphi}$ and $\beta \equiv S_{2} / M r^{2}$. In the case when the spin is equal to 0 (i.e. $\beta=0$ ), from equation (12) follows the known second-order equation relatively $u_{\perp}$ for the geodesic circular orbits together with the conclusion that such orbits exist at $r>3 m$ only. If $\beta \neq 0$, equation (12) can be used for analysis of the possible circular motions of a spinning particle.

First of all, we see that at $r=3 m$ equation (12) has the single solution

$$
u_{\perp}=-\frac{1}{3 \beta} .
$$

For the estimate of the absolute value $\left|u_{\perp}\right|$, in (10) we take into account the condition for a test spinning particle [18]

$$
\frac{\left|S_{0}\right|}{M m} \equiv \varepsilon \ll 1
$$

By equations (10), (13), (14) we have

$$
\left|u_{\perp}\right|=\frac{3^{1 / 4}}{\sqrt{\varepsilon}} .
$$

Hence $u_{\perp}^{2} \gg 1$, that is, for the motion on the circular orbit $r=3 m$, the velocity of a spinning particle must be ultrarelativistic, the more ultrarelativistic the smaller is the ratio of spin to mass of the particle.

Before the analysis of the solutions of equation (12) at $r \neq 3 m$, we stress the following important fact. If instead of equation (11) its shortened variant

$$
M \frac{D}{d s} u^{\lambda}=-\frac{1}{2} u^{\pi} S^{\rho \sigma} R_{\pi \rho \sigma}^{\lambda}
$$

is taken into account [Equation (16) is often considered in different papers], then for the description of the possible circular orbits of a spinning particle in a Schwarzschild field follows the equation

$$
u_{\perp}^{2}\left(1-\frac{3 m}{r}\right)-u_{\perp} \frac{3 m}{r} \beta-\frac{m}{r}=0 .
$$


Equation (17) at $r=3 m$ has the single solution $u_{\perp}=-1 / 3 \beta$ which coincides with (13). That is, the orbit $r=3 \mathrm{~m}$ is particular in the sense that the corresponding solution for $u_{\perp}$ is common for equations (12) and (17).

It is easy to see that according to (12) the circular ultrarelativistic orbits of a spinning particle exist in the small neighborhood of the value $r=3 m$ as well, as for $r>3 m$ and for $r<3 m$, in contrast with the geodesic circular orbits. In particular, if $r=3 m(1+\delta), \quad|\delta| \ll \varepsilon$, then instead of (13), (15) from (12) we obtain

$$
\begin{aligned}
& u_{\perp}=-\frac{1}{3 \beta}\left(1-\frac{3^{3 / 2}}{2} \frac{\delta}{\varepsilon}\right), \\
& \left|u_{\perp}\right|=\frac{3^{1 / 4}}{\sqrt{\varepsilon}}\left(1-\frac{3^{3 / 2}}{2} \frac{\delta}{\varepsilon}\right) .
\end{aligned}
$$

Here we stress that for the concrete orbit of radius $r=3 m(1+\delta)$ with $\delta>0$ the conditions of realization of the motion for the spinning test particle and the particle without spin are essentially different. Indeed, according to the geodesic equations, for the motion on such an orbit the particle must posses the velocity corresponding to the relativistic Lorentz factor of order $1 / \sqrt{\delta}$. Whereas, according to (19), for the motion on the orbit of the same radius at $0<\delta \ll \varepsilon$ the velocity of the spinning particle must correspond to the Lorentz factor of order $1 / \sqrt{\varepsilon}$. That is, the ratio of the Lorentz factor values for these cases is equal to $\sqrt{\varepsilon / \delta}$ and at very small $\delta$ becomes considerably greater than 1. Formally at $\delta=0$ this ratio is equal to $\infty$. Actually it means that a particle without spin and with nonzero mass of any velocity close to the velocity of light, starting in the tangential direction from the position $r=3 m$, will fall on the horizon surface within a finite proper time. On the other hand, a spinning particle will remain indefinitely on the circular orbit $r=3 m$ due to the interaction of its spin with the gravitational field. This interaction compensates the usual (geodesic) attraction. Certainly, this picture holds in the ideal case, when perturbations are neglected, because the circular orbits in the neighbor of $r=3 m(1+\delta)$ are unstable both for a particle without spin and for a spinning particle. In reality, one is dealing with fragments of trajectories close to the corresponding circular orbits.

We point out that equation (12) at $m=0$ has (beside the trivial case $u_{\perp}=0$ ) the solution

$$
u_{\perp}=\frac{1}{\beta}
$$

Relationship (20) describes the known Weyssenhoff circular orbits [19] of the non-proper center of mass ( by the terminology of C.Möller [20]) of a body rotating in the Minkowski spacetime. It is important that at the fixed sign of $S_{2}$ (i.e. when the direction of the inner rotation is fixed) the signs of $u_{\perp}$ in (18) and (20) are opposite. It means that the circular orbits in a Schwarzschild field which are described by equations (13), (15), (18), (19) do not pass into the Weyssenhoff orbits at $m \rightarrow 0$, and the physical nature of these orbits is different. This conclusion is also supported by the fact that the Weyssenhoff orbits correspond to equation (12) and do not correspond to equation (17), whereas 
solution (13) is common for equations (12) and (17). In this context we emphasize the following fact. Though equations (13), (18) describe the solutions of equations (11), (2) under condition (3), these solutions satisfy (in the main appoximation) condition (44) as well. Indeed, from (3), 6), (17) we have the nonzero components of $S^{\mu \nu}$ for a spinning particle on the circular orbits:

$$
S^{13}=-S^{31}=\frac{S_{2}}{r^{2}}, \quad S^{14}=-S^{41}=\frac{u_{3} S_{2}}{u_{4} r^{2}} .
$$

Taking into account expressions (15), (10), (13), (14) and (21) we obtain

$$
\begin{gathered}
P^{1}=0, \quad P^{2}=0, \\
P^{3}=-\frac{3^{-3 / 4} M}{m \sqrt{\varepsilon}}\left(1-\frac{11 \sqrt{3}}{18} \varepsilon\right) \text { sign } S_{0}, \\
P^{4}=\frac{3^{3 / 4} M}{\sqrt{\varepsilon}}\left(1+\frac{7}{6 \sqrt{3}} \varepsilon\right) .
\end{gathered}
$$

By equations (21), (22) it is easy to check that in this case

$$
\begin{gathered}
S^{2 \nu} P_{\nu}=0, \quad S^{3 \nu} P_{\nu}=0, \quad S^{4 \nu} P_{\nu}=0 \\
S^{1 \nu} P_{\nu}=\frac{1}{9} \varepsilon^{2} m M^{2} .
\end{gathered}
$$

So, the expression $S^{1 \nu} P_{\nu}$, though is not equal to 0 , is proportional to $\varepsilon^{2}$. We also stress that in the practical situations $m M^{2} \ll 1$ (for example, in the system used $m$ for the Sun is equal to $5 \cdot 10^{-6}$, and $M$ for an electron is equal to $2 \cdot 10^{-66}$ ). That is, the value of $S^{1 \nu} P_{\nu}$ is much less than 1 . In this sense the above considered solutions of equations (11), (2) determined by (13), (18) are practically common for conditions (3) and (41). It is known that under condition (41) equations (11), (2) do not have solutions of the Weyssenhoff type.

It is interesting that equation (12) has a single real solution for any fixed $r$ from the interval $2 m<r<3 m$ which is not located in the small neighbor of $r=3 m$. The corresponding dependence on $r$ is

$$
u_{\perp}=\frac{1}{\beta}\left(1-\frac{2 m}{r}\right)\left(1-\frac{3 m}{r}\right)^{-1}\left[1-\frac{3 m}{r}\left(1-\frac{2 m}{r}\right)^{-1} \beta^{2}\right]
$$

where, as above, we take into account the condition $\beta^{2} \ll 1$. That is, as in cases (13), (18), the value $u_{\perp}$ is ultrarelativistic. It is important that (because $1-3 m / r<0$ ) the sign of $u_{\perp}$ is opposite to the sign of $\beta$, as in cases (13), (18) and in contrast with the Wyessenhoff case (20). By this feature the circular orbits from the interval $2 m<r<3 m$, described by expression (24), can be interpreted as such whose nature is the same as for orbits (13), (18). Namely, these orbits are caused by the interaction of the spin with the gravitational field. According to the analysis from [21, for any (circular or not) motion of a spinning particle in a Schwarzschild field, this interaction has specific spin-orbit properties. At the same time, it is easy to see that equation (12) 
at $r>3 m$ has the solution for $u_{\perp}$ which at $m / r \rightarrow 0$ passes into (20), i.e. this solution is of the Weyssenhoff type.

The behaviour of a spinning test particle on circular orbits in the Schwarzschild and Kerr fields (mainly the clock effect) was studied in latest papers [22] at different conditions for the Mathisson-Papapetrou equations. Different types of orbits of the spinning particles in a Schwarzschild field were investigated in [23] on the base of the Mathisson-Papapetrou equations at condition (4).

\section{Numerical estimates and conclusions}

So, according to the Mathisson-Papapetrou equations a spinning test particle can moves on ultrarelativistic equatorial circular orbits with the values of the radial coordinate $r$ from the region near $r=3 m$ in a Schwarzschild field. The spin of a particle on these orbits is orthogonal to the motion plane, and, by (15), (19), the particle's velocity is more higher, the smaller is the ratio of spin to mass of the particle.

The existence of ultrarelativistic circular orbits of a spinning particle in a Schwarzschild field, which differ from geodesic circular orbits, probably, can be discovered in the synchrotron radiation of protons or electrons. Let us estimate the value $\varepsilon=\left|S_{0}\right| / M m$ for these particles in the cases when the Schwarzschild source is 1) a black hole of mass that is equal to three of the Sun's mass, and 2) a massive black hole of mass that is equal to $10^{8}$ of the Sun's mass. In the first case, taking into account the numerical values of $S_{0}, M, m$ in the system used, we have for protons and electrons correspondingly $\varepsilon_{p} \approx 2 \cdot 10^{-20}, \quad \varepsilon_{e} \approx 4 \cdot 10^{-17}$, whereas in the second case $\varepsilon_{p} \approx 7 \cdot 10^{-28}, \quad \varepsilon_{e} \approx 10^{-24}$. Because for the motion on above considered circular orbits the spinning particles must possess the velocity corresponding (according to equations (15), (19)) ) to relativistic Lorentz $\gamma$-factor of order $1 / \sqrt{\varepsilon}$, in the first case we obtain $\gamma_{p} \approx 7 \cdot 10^{9}, \quad \gamma_{e} \approx 2 \cdot 10^{8}$, and in the second case $\gamma_{p} \approx 4 \cdot 10^{13}, \quad \gamma_{e} \approx 10^{12}$.

As we see, in the case of a massive black hole the necessary values of $\gamma_{p}$ and $\gamma_{e}$ are too high even for extremely relativistic particles from the cosmic rays. Whereas if the Schwarzschild source is a black hole of mass that is equal to 3 of the Sun's mass, some particles may move on the circular orbits considered above. Analysis of a concrete model, closer to the reality, remains to be carried out. Here we point out that by the known general relationships for the electromagnetic synchrotron radiation in the case of protons or electrons on the considered circular orbits we obtain the values from the gamma-ray range. By the known features of the synchrotron radiation and the null geodesic trajectories, the part of the synchrotron radiation which arises from the orbits with $r<3 m$ cannot leave the surface of radius $r=3 m$ and will fall on the black hole.

It is known that he highly relativistic circular orbits of a spinless test particle are of importance in the classification of all possible geodesic orbits in a Schwarzschild field. The results of the present work show the significance of the ultrarelativistic circular orbits of a spinning particle for the description of all possible essentially nongeodesic trajectories following from the Mathisson-Papapetrou equations. First of all, namely 
on the circular or close to the circular ultrarelativistic orbits the spinning particle feels the maximal effect of the gravitational spin-orbit interaction. It would therefore be interesting to study the dynamics of the spinning particle deviating from the above considered circular orbits. This is our purpose for the next paper. Naturally, the problem of considerable importance is the search of the physical situations where the phenomena connected with different types of the ultrarelativistic motions of the spinning particles can be discovered. 


\section{References}

[1] Breuer R A, Chrzanowski P L, Hughes H G and Misner C W 1973 Phys. Rev. D 84309

[2] Chrzanowski P L and Misner C W 1974 Phys. Rev. D 101701

[3] Saijo M, Maeda K, Shibata M and Mino Y 1998 Phys. Rev. D 58064005

[4] Suzuki S and Maeda K 2000 Phys. Rev. D 61024005

[5] Mohseni M and Sepangi H 2000 Class. Quantum Grav. 174615

[6] Ellis J, Mavromatos N E, Nanopoulos D V and Sakharov A S 2003 Preprint astro-ph/0309144

[7] Mathisson M 1937 Acta Phys. Pol. 6163

[8] Papapetrou A 1937 Proc. R. Soc. A 209248

[9] Pirani F A E 1956 Acta Phys. Pol. 15389

[10] Tulczyjew W 1959 Acta Phys. Pol. 18393

[11] Dixon W 1970 Proc. R. Soc. A 314499

[12] Tod K P and de Felice F 1976 IL Nuovo Cimento 34365

[13] Semerak O 1999 Mon. Not. R. Astron. Soc. 308863

[14] Wong S 1972 Int. J. Theor. Phys. 5221

Kannenberg L 1977 Ann. Phys.(N.Y.) 10364

Catenacci R and Martellini M 1977 Lett. Nuovo Cimento 20282

Audretsch J 1981 J. Phys. A 14411

Gorbatsievich A 1986 Acta Phys. Pol. B 17111

Barut A and Pavsic M 1987 Class. Quantum Grav. 441

[15] Micoulaut R 1967 Z. Phys. 206394

[16] Carmeli M, Charach Ch and Kaye M 1977 Phys. Rev. D 151501

[17] Mashhoon B 1971 J. Math. Phys. 121075

[18] Wald R 1972 Phys. Rev. D 6406

[19] Weyssenhoff J and Raabe A 1947 Acta. Phys. Pol. 97

[20] Möller C 1949 Commun. Dublin Inst. Advan. Studies A 53 Möller C 1949 Ann. Inst. Henri Poincare 11251

[21] Plyatsko R, Bilaniuk O 2001 Class. Quantum Grav. 185187

[22] Bini D, de Felice F and Geralico A 2004 Preprint gr-qc/0410082

Bini D, de Felice F and Geralico A 2004 Preprint gr-qc/0410083

[23] Suzuki S and Maeda K 1997 Phys. Rev. D 554848 